\title{
Fighting the wicked problem of plastic pollution and its consequences for developing regions with expert and crowd solutions
}

\author{
Simon Fuger \\ University of Innsbruck \\ simon.fuger@uibk.ac.at \\ Moritz N. Lang \\ University of Innsbruck \\ moritz.lang@uibk.ac.at
}

\author{
Anna N. Köhl \\ University of Innsbruck \\ anna.koehl@student.uibk.ac.at \\ Johann Füller \\ University of Innsbruck \\ johann.fueller@uibk.ac.at
}

\author{
Thomas Ströhle \\ University of Innsbruck \\ thomas.stroehle@uibk.ac.at \\ Katja Hutter \\ University of Innsbruck \\ katja.hutter@uibk.ac.at
}

\begin{abstract}
The wicked problem of plastic pollution is one of the key global challenges. Finding adequate solutions to this complex problem requires cross-cultural and inter-organizational collaboration among diverse sets of stakeholders. In this context, the Ellen Mac Arthur Foundation approaches the problem of plastic pollution not only by involving experts into innovation processes but also by integrating the general public in form of an IT enabled crowdsourcing initiative. In this study, we analyze the outcomes of these actions with the help of automated text mining techniques. Our analysis demonstrates significant differences between the solutions given by experts and the crowd along various criteria. Further, this study provides guidance for practitioners on how to integrate diverse sets of individuals in problem solving processes with the help of information systems technologies. Especially for sustainability issues affecting both, developed and developing regions.
\end{abstract}

\section{Introduction}

One of the main environmental challenges in recent decades is the problem of plastic pollution. The main roots of the problem lie in western countries, whereas the consequences are severely felt in developing regions. In 2010, one person in the US created $124 \mathrm{~kg}$ of plastic waste per year, compared to only $3.6 \mathrm{~kg}$ in India [39]. Nevertheless, $86 \%$ of all ocean plastic derives from rivers in Asia, e.g. an estimate of 115,000 t of plastic enter the ocean through the Ganges River in India each year (Data from 2015, no more recent data available) [39]. Differences in waste management and global waste trade are among other things responsible for the pollution shift towards developing regions.
The ocean and beach pollution especially visible in developing regions generated worldwide awareness around this topic and lead to numerous cross-cultural initiatives where academia, politics and economy are desperately searching for solutions $[16,31,37,42,49$, 50]. However, effectively working solutions are few [28]. One of the reasons why it is so difficult to tame this problem is the wicked nature of plastic pollution, containing social, economic and environmental attributes [47]. For wicked problems no single best solution exists, rather a set of solutions is required to integrate different or even contrary perspectives, local circumstances and cross-cultural interdependencies $[10,17]$. To find viable solutions, the Ellen MacArthur Foundation (EMF) founded the New Plastics Economy (NPEC) Initiative in 2016. As part of the initiative, experts as well as the crowd were asked to find solutions to this problem.

Existing research highlights that experts and the public crowd may be valuable sources for solving complex problems [20] and for moving to a greener and more inclusive society $[9,10,21,32]$. An indefinite number of individuals contributing to problem solving via IT enabled crowdsourcing approaches are especially valuable to induce a holistic understanding and show different perspectives. Within IT enabled crowdsourcing initiatives, practitioners struggle to make use of the data generated, resulting in the consideration of only the winning ideas without utilizing the vast amount of all contributions $[6,27,30]$. In contrast to the open innovation community which focuses on the saying "diversity trumps ability" [8], traditional R\&D and innovation departments heavily rely on direct expert knowledge. Similar to the manual analysis of all crowd solutions, the comparison to the solutions of experts ties many resources and is therefore seldomly implemented in a systematic process. 
New technologies especially in the field of natural language processing allow to analyze numerous solutions efficiently without tying human resources. In this sense, we explore (1) if and how solutions from expert approaches differ from IT enabled crowdsourcing approaches and try to (2) explain how experts and the crowd contribute to solve wicked problems and sustainability issues through multi-level collaboration. To do so, we empirically compare two reports on plastic pollution written by leading experts and analysts with the outcomes of an IT enabled crosscultural crowdsourcing initiative focusing on idea generation in the context of plastic pollution.

We apply text mining techniques providing an immediate and holistic overview of content, as well as highlighting differences of expert and crowd documents based on relations of words. Differences are not only shown on a structural level, but also on a content level. In a content network analysis, we apply weights like the term frequency (tf) and the inverse document frequency (idf) for data wrangling. A second method, the topic modelling technique employing the Latent Dirichlet Allocation (LDA) is applied to strengthen our findings. This machine assisted multi-method approach leads to two main findings. First, we show that the solutions from experts and the crowd differs significantly in their nature highlighted along several criteria. The inclusion of various, diverse stakeholders through interorganizational and cross-cultural IT enabled approaches is beneficial to identify solutions for wicked problems and issues in developing regions where a magnitude of different ways of thinking is essential. Second, our findings lead to a roadmap aiding practitioners aiming at tackling a wicked problem with the help of multi-level collaboration.

\section{Literature Review}

\subsection{The wicked problem of plastic pollution}

The problem of plastic pollution has become a sustainability issue especially visible in developing regions creating globalized sensation and action. A vast number of initiatives is necessary, ranging from plastic avoidance over substitution to recycling methods and behavioral changes. The issue of plastic pollution can be seen as a wicked problem, as solutions need the involvement of cross-cultural stakeholders in a "collaborative problem solving and iterative process" [11] and due to its inextricable mix of social, economic, and environmental attributes [47]. Wicked problems are defined by common characteristics, which indicate the difficulty to define and solve such problems. There is no single right solution but only a set of numerous solutions [40]. Also, wicked problems are interconnected and often symptoms of other problems.

Regarding the issue of plastic pollution, economic interest of multinational companies in the chemical, packaging and food industry stand against complex and contrary environmental and social issues. Plastics have positive characteristics, e.g. in regard of longevity of food or low weight in logistics. Nevertheless, there are various negative side effects, e.g. due to the toxicity of the material or the issue of micro-plastics. Especially the global disparity regarding waste management possibilities and global waste transactions are critical issues. Whereas western countries are the biggest producers of plastic waste per person [39], developing regions are often the victim of plastic pollution. Due to trade off from western plastic waste to countries without legal waste management systems, people in developing regions experience plastic pollution in water ways and beaches firsthand.

Therefore, the problem-solving process of wicked problems should support the development of solutions that are cross-cultural, integrative and contextualized [10]. The success of solutions is strongly dependent on the context and local circumstances, as the example of bioplastic shows [12, 14]. In countries with functioning waste management systems, biodegradable plastics have the undesirable side effect of influencing the existing recycling stream of conventional plastics [37, 49]. In developing regions without existing waste management systems, where plastic leaks directly into the environment, biodegradable plastics can be a solution. In this situation the polymer structure can degrade back into natural materials.

Solution approaches to plastic pollution need to consider inter-organizational and cross-cultural stakeholder involvement, differing circumstances, complex material properties, biological interactions, resonance in society and interaction with economic objectives [48]. On the one side, deep expert knowledge is needed to find viable chemical and economic solutions on how to recycle plastics, on the other side, the exchange of different, often contrary perspectives of a diverse group of individuals in multistakeholder ecosystems is essential $[10,14,24,29$, 35].

Next to the traditional approach of expert and scientific solution approaches, (e.g. expert reports), IT enabled open innovation formats such as crowdsourcing have become increasingly popular to tackle sustainability and development issues [43]. They are characterized by the described diversity. 
Such formats help to actively involve stakeholders affected by the problem [29].

\subsection{Crowdsourcing as a promising tool to tame plastic pollution}

Lately, research in the field of open and distributed innovation has shown that crowdsourcing was reinforced as a promising tool to find viable solutions at low costs and higher performance, especially for complex problems where existing innovation departments had no solution yet $[1,25]$. The collaboration among key stakeholders can be supported by creating specific platforms and facilitated processes to encourage the dialogue and cocreation of activities.

The power of crowdsourcing was previously shown in various ways by renowned companies such as BASF, Siemens or Procter and Gamble. Examples range from the collaborative content creation at Wikipedia [6], to open source projects such as Linux [7], or idea competitions such as the NASA ISS Longeron challenge [38]. Such crowdsourcing innovation examples show that IT enabled cooperation and knowledge sharing among heterogeneous participants provides venues to find solutions to challenging problems $[6,19]$.

Various studies have shown that a large diverse crowd of independent and unknown individuals performs better on certain types of challenges than a small number of experts. There is no clear elaboration in existing literature on which types of challenges are better tackled with crowdsourcing techniques. The underlying theoretical foundation of crowdsourcing for innovation is the value of knowledge and expertise diversity referring to "diversity trumps ability" [8]. The greater the size of the crowd, the greater the possibility of idea diversity and the greater the probability of novel ideas and approaches [25, 30]. While quite substantive research exists that shows the potential of crowdsourcing most of the studies focus on the single best solutions. We argue that there is much more value in IT enabled collaboration initiatives than solely focusing on a few winning ideas. Research so far mainly explored how to find the single best solutions among all contributions [6, 30]. The added value of the multiple ideas generated within a crowdsourcing initiative is often neglected due to the effort necessary to read and analyze the vast amount of ideas [27]. Traditionally, unstructured data is interpreted using qualitative data approaches, such as reading and manual coding, but given the size of data sets obtained any kind of manual analysis is virtually impracticable [33]. As an example, Google's project
10100 can be mentioned where over 150,000 submissions were collected which needed a team of 3,000 internal employees to evaluate the generated content [23]. Most of the time the expected outputs do not justify such enormous efforts. However, especially for wicked problems such as plastic pollution a broad understanding is valuable in order to better understand the multiple perspectives from different stakeholders and to produce effective solutions $[10,11,14,40]$.

There is only limited knowledge about how the ideas differ in terms of content and solution approaches. This can be described as a black box in open innovation research. If researchers were able to identify novel content within all contributions of a IT enabled crowdsourcing initiative, practitioners would benefit on a whole different level compared to only focus on winning ideas [26].

\section{Methodology}

\subsection{Case description}

To explore if and how outcomes from experts differ from the crowd, we empirically compare the outcomes of a crowdsourcing initiative focusing on plastic pollution solutions with the reports written by experts in this area, published within the same research initiative.

We focus on actions taken by the Ellen MacArthur Foundation (EMF) in the scope of the New Plastics Economy (NPEC) Initiative. It was founded in 2016 with the goal to tackle the current plastic pollution problem. For this study, we concentrate on two sets of data from two different initiatives within NPEC: (1) two reports published in 2016 [49] and 2017 [50] and (2) an IT enabled crowdsourcing initiative conducted in 2017 [13]. Both initiatives were set-up having the same goal in mind, tackling the current plastic pollution problem, and finding adequate solutions also for developing regions.

Authors of the reports were the EMF Team Members of the NPEC Initiative with analytical support of McKinsey (Report 1) and Systemiq Ltd. (Report 2). The authors of both reports are proven experts in the areas of circular economy, international policy and regulations, business and economics, chemistry, and biology. The insights described in the two reports are the results of a detailed segment-bysegment analysis of the plastics landscape. The analysis is based on existing research, expert interviews, and the insights from the strategic partners of EMF, e.g. Coca-Cola and Unilever. The interviews include experts from waste management systems, 
producer responsibility organizations, the chemical industry, the packaging industry, the food industry as well as research institutes. What opposes the diversity of the experts to the diversity of a crowd is their common high level of education and professional standing.

To extend the expert reports, an IT enabled crowdsourcing initiative was launched by EMF on OpenIDEO to integrate a diverse group of individuals into the discussion. OpenIDEO is a platform with a community of over 17,000 users from 170 different countries. The initiative of interest hosted by EMF on this platform is called Circular Design Challenge (CDC) and tackles the question of how to get products to people without generating plastic waste [13]. The CDC was launched in May 2017 and over a period of six months participants submitted their solutions to tackle the problem of plastic waste. Participation was open to everybody. In total, 1107 users took part in the competition posting ideas, commenting, and liking them. Overall, 619 ideas were submitted by 483 individuals and in the end 16 ideas were evaluated by an expert jury as winning ideas in different categories. The crowd was spread around the world, coming from 64 different countries including participants from developing regions such as India or central African countries. Profiles of the participating individuals revealed a total of 171 different occupations, ranging from architects, over engineers, wildlife experts, social workers, to graduate students.

\subsection{Method}

To analyze the vast amount of texts from different sources we use automated text mining techniques to understand the solution spaces of the content from the reports and the ideas by the crowd. To distinguish the content of different text corpora [44] we apply two approaches, content network analysis with the use of the term frequency - inverse document frequency (tfidf) [41] and topic modelling by a Latent Dirichlet Allocation (LDA) [5]. Both methods are used to gain a better understanding of the content as well as similarities and differences. To assure a correct analysis of these solution spaces and the interrelatedness of such, an expert in the field of plastic pollution co-authored this article with special focus on the interpretation of the results. All computations were performed using $\mathrm{R}$ version 3.5.2 [36].

As a starting point, data is collected and preprocessed for analysis. Data is derived from two different sources: (1) the two EMF reports and (2) the ideas of the CDC on OpenIDEO. The EMF reports are publicly available at the NPEC homepage. Report 1 (document 1) consists of a total of 59,372 words, and report 2 (document 2) comprises 26,099 words. To retrieve all ideas generated in the $\mathrm{CDC}$ on OpenIDEO (document 3) a web crawler was used. The data was fetched in October 2017 and a corpus combining all ideas in a single text string was formed. This single "document" consists in total of 219,244 words. There are significant differences in the total number of words per document. However, as the employed methodologies only make use of relative frequencies of the respective vocabularies, the differences in the absolute number of words per document have no implications on the applied statistical analysis. Moreover, it was tested that the different vocabularies have a sufficient percentage of intersecting number of words between the three documents. In a first automated text cleaning step, differences in the text structure of the three documents are eliminated. All stop words (e.g. "a", "by", "the"), punctuations or digits (e.g. "hyperlink", "date", "page number"), words with a character size under three and nonstandard UTF-8 characters are removed. In a final preprocessing step, all words were stemmed by reducing the inflected words to their word stem.

The content network analysis with the use of the tfidf makes use of a combination of a pairwise count within sections and the tf-idf weight. We use the pairwise counts [44] as a measure of the individual word connections. The tf-idf counts the number of times each pair of words appears together within a predefined segment of consecutive words and is calculated rolling over all segments in the document. E.g. for an arbitrary combination of two words in one segment of a pre-defined number of consecutive words the counter of this pair will count plus one. For this study, an equidistant segmentation size of ten words is chosen, which corresponds to the typical length of a single sentence. As the algorithm also finds word connections between common bigrams (e.g. "stainless steel") and trigrams, these are automatically removed from the documents beforehand.

The inverse document frequency (idf), which is part of the tf-idf, is a measure of how common or rare a word is across all documents. It can be used for filtering and sorting the data to obtain relevant characteristics. Let $D$ be the set of all documents, $N=$

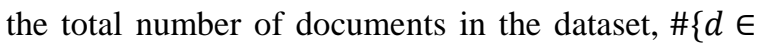
$D: w \in d\}$ the number of documents where the word $W$ appears, then the idf is given as follows [41]: $\operatorname{id} f(w, D)=\log \frac{\# D}{\#\{d \in D: w \in d\}}$. The idf can be interpreted as a weight to influence the wordfrequency. In our special case of having three 
documents, the idf has the following characteristics: (1) idf $=0$, if a word or a word pair exists in all three documents, (2) idf $\sim 0.4$, if a word exists in two documents and (3) $i d f \sim 1.1$, if a word is unique within a single document. Additionally, the termfrequency(tf) is the relative frequency of a word in its document and given as follows: $t f(w, d)=$ $\frac{\#(w, d)}{\sum_{t \in d} \#(t, d)}$, where \#(w,d) gives the frequency of a word $w$ in a document $d$. Every word or word pair has a separate tf for each document $d$. Both weights, the idf and the tf, can be combined in the tf-idf and is given as follows : $t f$-idf $(w, d, D)=t f(w, d) *$ $i d f(w, D)$

In this study, the tf-idf is used to omit words which have a low term frequency. A network visualization is employed to show the most relevant differences and similarities of the documents for the content network analysis. To illustrate the frequency of all words or connections, the font size within a node is shown proportionally to the term-frequency in its document and the width of an edge is shown proportionally to its appearance in pairs, which is given through the previously determined pairwise count. The idf or the tf-idf weight is used to filter the relevant edges and nodes. The network visualization is implemented via the package vis.js [2].

The second method applied makes use of the LDA, which is a generative statistical model that allows sets of words to be explained by a small number of unobserved topics. In an iterative process each word is attributed to one of the document's topics. Hence, it reveals the hidden relations between topics, meaning that it can also be employed to show similarities and differences between documents [5].

The LDA procedure is executed by an implementation of the package topicmodels [22] and needs a predefined number of topics. Two metrics defined by Arun et al. [4] and Deveaud et al. [15] are analyzed for a number of topics ranging from 2 to 18 . For a better comparison both metrics are normalized from 0 to 1 . The Arun et al. [4] metric, which should be maximized, suggests three to four topics. The Deveaud et al. [15] metric suggests three to six topics. To prevent overfitting without performance loss and to keep the interpretation of the topics as simple as possible, three topics are chosen for the applied LDA algorithm within this study. The LDA procedure is performed by a Gibbs sampling, which returns only the best model with respect to the posterior likelihood. A burn-in of 1000 iterations and recording every 50th iteration for 2000 iterations are used as parameters.

\section{Results}

For the remainder of this article, we use the word stems shown in the visualization and list the most common un-stemmed words in brackets for a better understanding. On a random basis we compared the results of the analysis with the original texts for congruence.

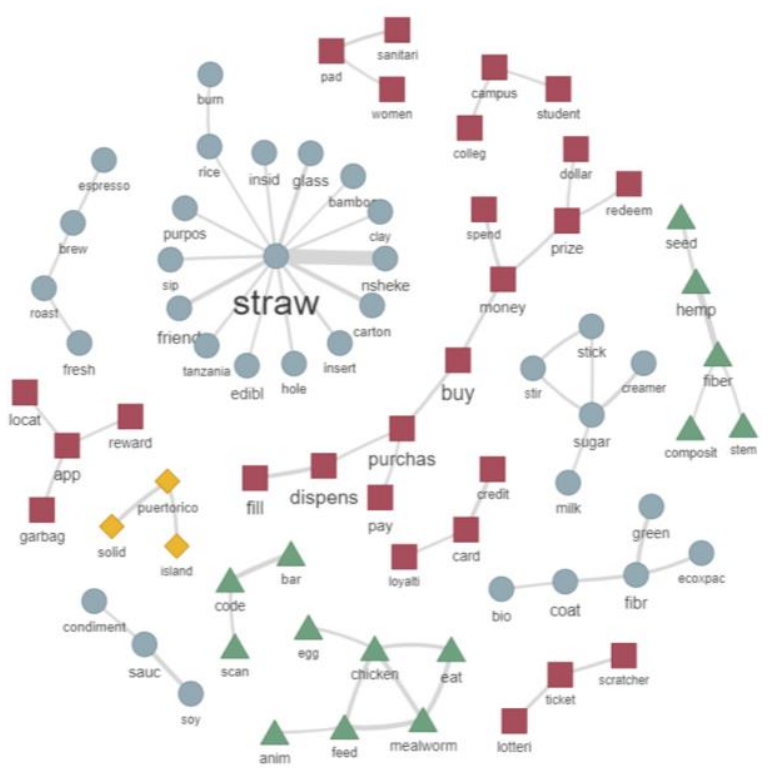

Figure 1. The network shows the connections representative for the CDC

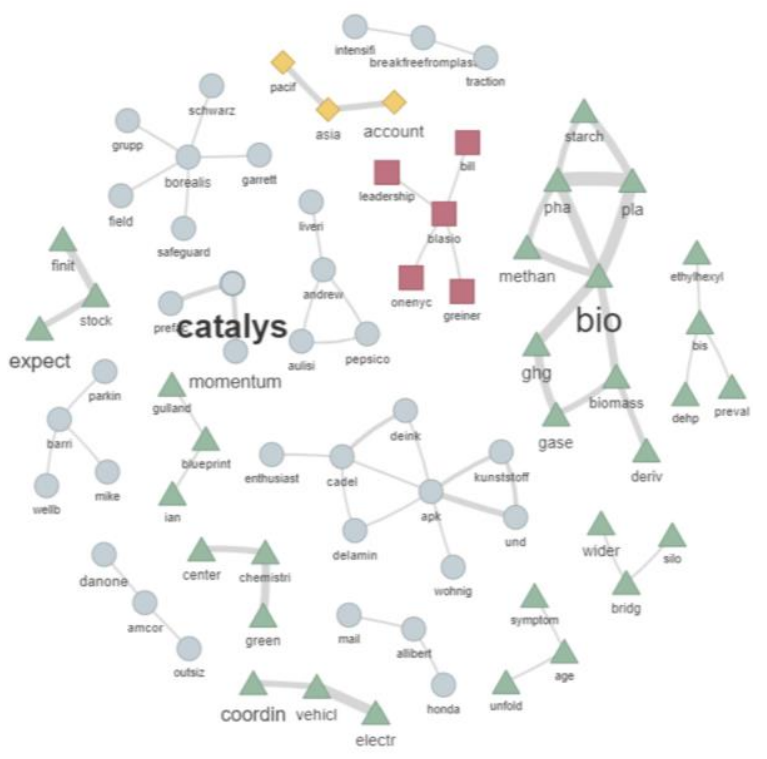

Figure 2. The network shows the connections representative for the two reports 
Figure 1 and 2 show two networks based on the tfidf analysis. Figure 1 illustrates the first 70 strongest connections for the IT enabled CDC based on the pairwise count frequency. Figure 2 is the counterpart of Figure 1 and shows the strongest connections for the two expert reports. The strongest connections representative for the $\mathrm{CDC}$ exhibit clear distinct themes not interconnected with each other. These themes, shaped by the word connections and frequencies are either connected through (1) a product (blue circle), (2) end users and their behavior (red square), (3) a region (yellow diamond) or (4) a specific technology (green triangle). The dominant theme in the middle forms around the product straw. Plastic straws as marine debris and as a threat to wildlife have gained significant media attention in recent years [16, 42].

This specific problem is picked up by the crowd and various alternatives are introduced, mostly in form of other materials such as glass, carton, bamboo, or Nsheke. We investigated the competition to understand the strong connection between the word stem straw and Nsheke and found that Nsheke is a naturally growing grass in the developing region of Tanzania, which is biodegradable and eco-friendly [34]. A theme around user behavior are the word stems loyalti (loyalties, loyalty,...), card and credit. This refers to giving incentives to customers for bringing back or recycling packaging materials. The theme around the word stem puertorico displays that the crowd also refers to local circumstances, and in this case the differences of plastic pollution between an island and mainland. A theme referring to a specific technology is around the word stem mealworm. In 2015 researchers from Stanford University found out that mealworms can safely digest Polystyrene in a way that is non-hazardous to their health and without generating toxic outcomes [46]. The crowd picked this scientific insight and generated possible business ideas around it, e.g. through feeding the mealworm to chicken, birds, insects etc. (e.g. [18]).

The strongest connections representative for the reports, displayed in Figure 2 also show clearly separated themes. Nevertheless, the themes are connected through a different logic than the themes of the CDC: a connection through (1) firms or initiatives (light blue circle), (2) specific technologies or materials (light green triangle), (3) regions (light yellow diamond) and (4) politics (light red square). Many small themes form around companies and/or initiatives. The companies mentioned are from different areas, e.g. the FMCG industry (danone and pepsiCo) or the packaging industry (amcor and allibert). In addition, also global initiatives, e.g. the \#breakfreefromplastic movement, a movement that narrates a positive story of how a future without plastics can look like, are mentioned. A look into the report approves these topics, as a strong focus on companies can be easily identified. The themes formed around these companies are often very specific, e.g. the theme around allibert refers to reusable packaging which is more environmentally and economically beneficial versus single-use cardboard boxes. Next to companies and initiatives, themes are formed around specific technologies and materials. The most frequent word stem is bio with strong connections to pha (Polyhydroxyalkanoates), pla (polylactide) and ghg (greenhouse gases). Pha and pla are both polyesters derived from renewable biomass, typically through microorganisms, e.g. bacterial fermentation of plant starch. Both polymers are biodegradable and are of the highest consumption volumes of any bioplastics in the world [45]. This also explains the other connections to this theme: starch, methan and biomass. Also, political regulations and acts are part of the two reports, as the theme with the word stems blasio, leadership and onenyc shows. Bill de Blasio, Major of New York City fought to ban all single use plastics [31].

In addition to the content network analysis, the visualizations in Figure 3 show the results of the applied topic modelling. It is conducted by an LDA implementation of Grün and Hornik [22] introduced in Section 3.2. The bar plots given in Figure 3 show the four most relevant words for each topic which are sorted by the logarithmized parameters of the word distribution. Figure 4 shows a heat map illustrating the assignment of topics per document. The visualizations are inspired by Silge and Robinson [44].

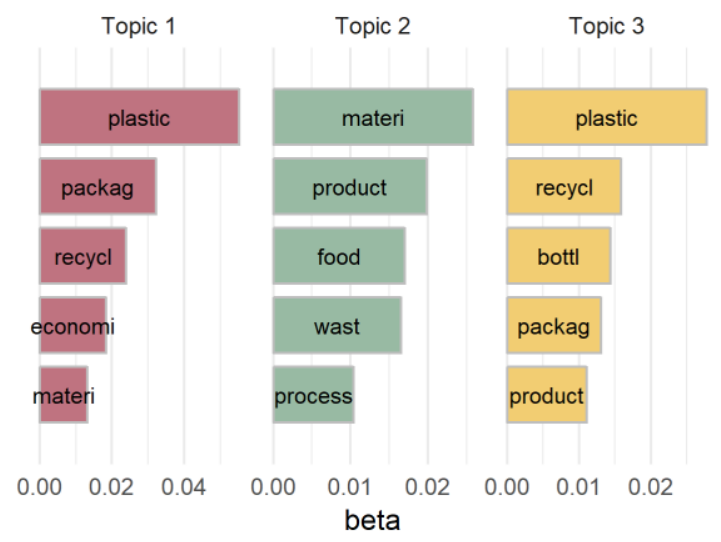

Figure 3. The most relevant words for each topic are shown as bar plots based on the applied topic modelling 
The heat map in Figure 4 is based on the different topics shown in Figure 3 and can be read as follows. Report 1 consists to $69 \%$ of topic 1, 29\% of topic 2 and $2 \%$ of topic 3 . In total, the row sum of every document totalizes in $100 \%$. The figure shows that the two reports mostly consist of topic 1 , whereas the CDC mostly consists of topic 3 . It is visible, that topic 1 is hardly present in the CDC (with $0,4 \%$ ) whereas topic 3 is hardly present in the two reports (with $0,2 \%$ and $0,4 \%)$.

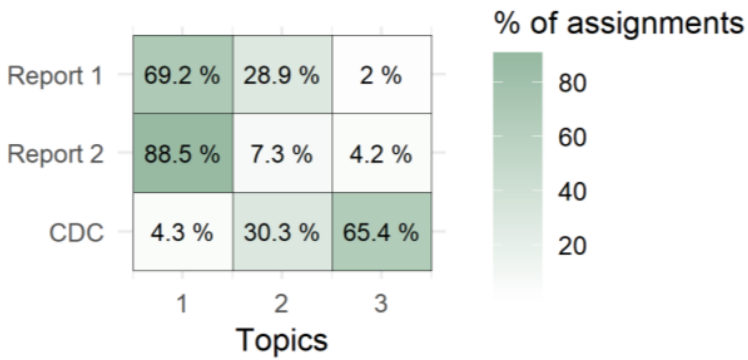

\section{Figure 4. The assignment of the topics per document is shown in the heatmap}

Topic 2 is not decisive in any of the three documents but can be seen as the connecting link between the reports and the CDC with word stems like materi (material,...) product, wast (waste, wasting,...), process, environ (environment, environmental,...) and develop (development, developing,...). The word stems plastic, packag (package, packaging,...) and recycl (recycle, recycling,...) are within the four most frequent words in topic 1 and 3 , but these are almost the only intersections between these topics. Topic 1, decisive for the two reports is set together of word stems indicating a global and economic perspective, e.g. economi/econom (economy, economies, economical,...), system, global, innov (innovation, innovative,...), design and market. Topic 2, determining the $\mathrm{CDC}$ is set together of word stems indicating a product and consumer-oriented perspective, e.g. bottl (bottle,...), product, cup, straw, consum (consumer, consuming,...), coffee, cap and people. The tendency that the crowd focuses more on specific circumstances such as products and end users is in line with the results from the tf-idf analysis. In addition, this analysis endorses the findings of the tfidf approach that the two reports are not focusing on specific products or end users, but rather on industries, companies, and systems.

\section{Discussion}

The empirical investigation allows this study to answer the posed research questions, namely (1) if and how solutions from expert approaches differ from IT enabled crowdsourcing approaches and (2) explain how experts and the crowd contribute to solve wicked problems and sustainability issues. The first question is addressed through a comparison of the expert and crowdsourcing outcomes along various criteria, highlighted in section 5.1. To answer the second research question, a roadmap is presented to guide practitioners and researchers to solve wicked problems such as sustainability issues in developing regions with the help of experts and crowd initiatives.

\subsection{Differences between expert and crowd solutions}

With the help of advancements in applicability of assisting technologies for data analytics, especially in the field of machine learning and natural language processing, this study aims to differentiate between the solution types given by experts and IT enabled approaches. This section provides a comparison based on differences in the environment the experts and the openIDEO participants are acting within.

5.1.1. Context and living environment. Most users of IT enabled crowdsourcing initiatives are participating in their leisure time, from their private computers, cell phones or through workshops organized within the community as part of their social live besides their regular job [3]. As shown in this study, participants were highly diverse in terms of their cultural milieu, occupation, and country of origin. A lot of participants come from developing regions and were able to address needs experienced in their day to day live. Their context is their living environment, with everyday live and work including their social environment. The problem of plastic pollution is associated with their personal context and with the plastic problems they either experience personally or which are especially apparent in their communities. This study is able to present this contextualization by the number of ideas around enduser products like straw and ideas changing consumer behavior (see Figure 1) and the most common words in Topic 3 (see Figure 3) which are particularly about reducing waste and using recyclables for everyday products. This contextualization is also apparent for the experts, only is the context a different one. This study reveals that the ideas of experts are mostly 
around technologies and companies and their possibility to scale to a global level (see Figure 2).

5.1.2. Geographical orientation. The second differentiation between the solutions given by experts and the crowd is their geographical focus. Figure 1 and 2 show that both subjects of analysis have a focus on geographic specifications (yellow diamond). Topic modelling nicely displays present differences in their geographical orientation. Topic 1 which is mainly prevalent in the two reports indicates a more system level, economy wide and global approach. The companies mentioned in the expert reports, e.g. Danone or Pepsi often have a global scope, whereas the ideas from the crowd often focus on local circumstances, as the example of Nsheke straws, grown in a developing region of Tanzania, shows. This goes in line with the contrasting context of crowd and experts.

5.1.3. Technology/feasibility. Both, experts, and crowd form considerable clusters around technological solutions (see Figure 1 and 2). A deeper analysis of the terms used by both and a look at the original texts reveals distinctions despite the apparent similarity. Experts predominantly use the technical terms, e.g. Polyhydroxyalkanoates or polylactide, relating to novel bioplastics derived through mechanisms of degradation, whereas terms used by the crowd are rather colloquial, as the mealworm example shows. It is more likely, that the crowd picks up existing technologies as a basis for novel business ideas and quick implementation possibilities. Experts tend to describe complex scientific processes and breakthroughs as part of their professional life.

In a similar vein, one can argue that the solutions proposed by the crowd tend to be easier and faster to implement. Screening the ideas presented, they most often focus on regional initiatives in need for support in terms of monetary incentives, community effort or knowhow in a specific field. In contrast, solutions proposed by experts most often involve systemic change including multiple stakeholders with different intentions and variant schedules. The solutions proposed by experts operate in a tremendously different scale regarding impact. Expert solutions of the two EMF reports most often have the advantage that when implemented they affect whole systems on a global scale. In contrast, the crowd solutions often lack replicability in different ecosystems/regions.

\subsection{Roadmap towards the inclusive use of expert and crowd solutions}

Our findings reveal differences in expert and crowd solutions. A challenge for practitioners from various fields is the choice of the right innovation practice for different cases of application. Focusing on idea generation in the field of wicked problems with the inclusion of industry experts on the one hand and the wisdom of the crowd on the other hand, this study provides guidance on the usage of such practices.

The combination of experts and the crowd helps to tackle wicked problems in a broad manner. Our findings suggest that the different approaches do not replace, but rather complement each other. Wicked problems also in developing regions are not solved through one global solution, just as little as they are solved through some local solutions [40].

There is an interdependency between local ideas for developing regions proposed by the crowd and global solutions of experts, and they can complement each other. Especially in the context of plastic pollution, which is an issue with its source in western countries but its negative effects occurring most severely in developing regions. Solely implementing local solutions in developing regions is not solving the root of the problem. Having multiple stakeholders and approaches entering problem solving processes is essential to find adequate and effective solutions to complex wicked problems [1, 25, 40].

For wicked problems no single best solution exists, rather a set of solutions is required to integrate different or even contrary perspectives and local circumstances $[10,17]$. Consequently, in applying a combination of expert knowledge and IT enabled crowd intelligence, practitioners are receiving the best solutions of both worlds to solve the wicked problem of plastic pollution and treat the root of the problem in western countries as well as treating the symptoms visible in developed regions. In practice however, it is more often a question of choosing one approach rather than implementing both approaches. Due to resource constraint (budget, time, human resources), a simultaneous implementation of expert and crowd approaches may not be possible. Based on the differences evaluated for research question one, this study helps to make a more profound decision.

Practitioners seeking to understand the magnitude of the problem and to unveil coherencies as a basis for the solution process are advised to choose expert integration. The knowledge and experience of experts in grasping complex and global interrelations is beneficial for the finding of high-level solution 
approaches. However, this study suggests that it may be difficult to break down these solutions to local circumstances and user behavior and may therefore require more systemic change on global level and longer implementation times.

Practitioners pursuing relatively tangible and easy to implement solutions to local circumstances in developing regions and consumer behavior in western countries are advised to integrate the knowledge of a large and indefinite crowd of individuals with the help of IT. Through this the development of solutions that are integrative and contextualized are supported, as supposed by Brunswicker et al. [10]. The solution to the problem may not lie in the global upscaling of one single idea, as their success is often bound to local circumstances, but may lie in the simultaneous implementation of a vast amount of different solutions in local context around the globe.

\section{Conclusion}

We contribute to the theory of wicked problems, by analyzing the content of two expert reports and a crowdsourcing initiative. To prove differences of these approaches, we use content network analysis and topic modelling approaches. Topic related conclusions were drawn based on the differences of content generated by a variety of stakeholders. Revealing an overview about the solutions proposed by both, experts and the crowd helps to understand the magnitude of perspectives the problem of plastic pollution can be tackled with. This study demonstrates that various and contextualized solutions are particularly found through the inclusion of different stakeholders.

Our research adds to the literature of open and distributed innovation and problem-solving $[1,25]$. So far, studies mainly focused on structural characteristics of crowdsourcing initiatives, analyzing the behavior of users, the influence of different reward systems or solely the qualities of winning ideas itself. We draw attention through analyzing such initiatives on a content level. Not only do more data points provide a better overview about the solutions proposed, but also the interrelatedness of such becomes more apparent. Our empirical results suggest that a content network analysis can lead to a more nuanced and fruitful understanding of text documents that focus on solving the complex wicked problem of plastic pollution. Therefore, we contribute by making use of big data analysis to identify most prominent topics and present a better understanding of a complex problem based on these outcomes.
Gathering diverse solutions is only the first step of tackling the problem itself. If stakeholders, such as governments, policy makers, companies, or NGOs, are not able to summarize, illustrate, communicate, and implement the proposed solutions a problem of this magnitude will remain unsolved. We propose a methodology that not only reveals insights in the generated content, but also enables practitioners to share information in a condensed way. The real benefit of the IT enabled challenges does not come from supporting 16 winning ideas but results from content generated by 1,107 individuals with diverse backgrounds also from developing regions collaborating on a matter that affects all of humanity. This is the result that needs to be distributed by challenge initiators to raise awareness and create momentum.

\section{References}

[1] Afuah, A. and C.L. Tucci, "Crowdsourcing As a Solution to Distant Search", Advanced Materials Research, 37(3), 2012, pp. 355-375.

[2] Almende, B.V. and B. Thieurmel, "Robert T. visNetwork: Network Visualization using 'vis. js' Library, R package version 2.0. 4. 2018", 2018.

[3] https://www.openideo.com/approach , accessed 7-1-2020.

[4] Arun, R., V. Suresh, C.V. Madhavan, and M.N. Murthy, eds., On finding the natural number of topics with latent dirichlet allocation: Some observations, Springer, 2010.

[5] Blei, D., A. Ng, and M.I. Jordan, "Latent Dirichlet allocation", Journal of Machine Learning Research, 3, 2003, pp. 9991022.

[6] Boudreau, K.J. and K.R. Lakhani, "Using the crowd as an innovation partner", Harvard Business Review, 91(4), 2013, pp. 60-69.

[7] Brabham, D.C., "Crowdsourcing as a model for problem solving: An introduction and cases", Convergence, 14(1), 2008, pp. 75-90.

[8] Brabham, D.C., Crowdsourcing, Mit Press, 2013.

[9] Brooks, S., X. Wang, and S. Sarker, "Unpacking green IS: a review of the existing literature and directions for the future", in Green business process management. 2012. Springer.

[10] Brunswicker, S., V. Bilgram, and J. Fueller, "Taming wicked civic challenges with an innovative crowd", Business Horizons, 60(2), 2017, pp. 167-177.

[11] Caiado, R.G.G., W. Leal Filho, O.L.G. Quelhas, de Mattos Nascimento, Daniel Luiz, and L.V. Ávila, "A literaturebased review on potentials and constraints in the implementation of the sustainable development goals", Journal of Cleaner Production, 198, 2018, pp. 1276-1288.

[12] Camillus, J.C., "Strategy as a wicked problem", Harvard Business Review, 86(5), 2008, p. 98.

[13] https://challenges.openideo.com/challenge/circular-design/ brief, accessed 5-1-2019.

[14] Conklin, J., "Dialogue mapping", Building Shared Understanding of Wicked Problems. West Sussex, England: John Wiley \& Sons, 2006. 
[15] Deveaud, R., E. SanJuan, and P. Bellot, "Accurate and effective latent concept modeling for ad hoc information retrieval", Document numérique, 17(1), 2014, pp. 61-84.

[16] Eagle, L., M. Hamann, and D.R. Low, "The role of social marketing, marine turtles and sustainable tourism in reducing plastic pollution", Marine pollution bulletin, 107(1), 2016, pp. 324-332.

[17] Eriksen, M., M. Thiel, M. Prindiville, and T. Kiessling, "Microplastic: what are the solutions", Freshwater Microplastics, 2018, p. 273.

[18] https://challenges.openideo.com/challenge/circular-design/ ideas/feeding-mealworms-polystyrene, accessed 4-23-2019.

[19] Füller, J., K. Hutter, and R. Faullant, "Why co-creation experience matters? Creative experience and its impact on the quantity and quality of creative contributions", R\&D Management, 41(3), 2011, pp. 259-273.

[20] Füller, J., K. Hutter, J. Hautz, and K. Matzler, "User Roles and Contributions in Innovation-Contest Communities", Journal of Management Information Systems, 31(1), 2014, pp. 273-308.

[21] Gellers, J.C., "Crowdsourcing global governance: sustainable development goals, civil society, and the pursuit of democratic legitimacy", International Environmental Agreements: Politics, Law and Economics, 16(3), 2016, pp. 415-432.

[22] Grün, B. and K. Hornik, "topicmodels: an R package for fitting topic models", Journal of Statistical Software, 13(40), 2011.

[23] Haller, J.B., K. Hutter, J. Füller, and K.M. Möslein, "Play or Vote: Matching Games as New Approach for Design Evaluation in Innovation Contests.", in Handbook of research on serious games as educational, business and research tools, M.M. Cruz-Cunha, Editor. 2012. IGI Global (701 E. Chocolate Avenue Hershey Pennsylvania 17033 USA): Hershey, $\mathrm{Pa}$.

[24] Innes, J.E. and D.E. Booher, Planning with complexity: An introduction to collaborative rationality for public policy, Routledge, 2010.

[25] Jeppesen, L.B. and K.R. Lakhani, "Marginality and problemsolving effectiveness in broadcast search", Organization Science, 21(5), 2010, pp. 1016-1033.

[26] Kakatkar, C., J. de Groote, J. Füller, and M. Spann, "Essay 2: The DNA of Winning Ideas-A Network Perspective of Success in New", Advanced Applications of Network Analysis in Marketing Science, 7, 2018, p. 49.

[27] Köhl, A., S. Fuger, M. Lang, J. Füller, and M. Stuchtey, "How Text Mining Algorithms for Crowdsourcing Can Help Us to Identify Today's Pressing Societal Issues", Annual Hawaii International Conference on System Science, 2019.

[28] Kramm, J. and C. Völker, "Understanding the Risks of Microplastics: A Social-Ecological Risk Perspective", in Freshwater Microplastics. 2018. Springer, Cham.

[29] Kunz, W. and H.W.J. Rittel, Issues as elements of information systems, Citeseer, 1970.

[30] Lakhani, K.R., K.J. Boudreau, P.-R. Loh, L. Backstrom, C. Baldwin, E. Lonstein, M. Lydon, A. MacCormack, R.A. Arnaout, and E.C. Guinan, "Prize-based contests can provide solutions to computational biology problems", Nature biotechnology, 31(2), 2013, p. 108.

[31] Merrington, A., "Recycling of plastics", in Applied plastics engineering handbook. 2017. Elsevier.
[32] Michelucci, P. and J.L. Dickinson, "The power of crowds", Science (New York, N.Y.), 351(6268), 2016, pp. 32-33.

[33] Müller, O., I. Junglas, J. Vom Brocke, and S. Debortoli, "Utilizing big data analytics for information systems research: challenges, promises and guidelines", European Journal of Information Systems, 25(4), 2016, pp. 289-302.

[34] https://challenges.openideo.com/challenge/circular-design/ top-ideas/nsheke-straws, accessed 2-5-2019.

[35] Ongondo, F.O., I.D. Williams, J. Dietrich, and C. Carroll, "ICT reuse in socio-economic enterprises", Waste management (New York), 33(12), 2013, pp. 2600-2606.

[36] R Core Team, "A language and environment for statistical computing. Vienna, Austria: R Foundation for Statistical Computing", http://www.R-project.org, 2018.

[37] Ren, X., "Biodegradable plastics: a solution or a challenge?", Journal of Cleaner Production, 11(1), 2003, pp. 27-40.

[38] Richard, E.E., J.R. Davis, J.H. Paik, and K.R. Lakhani, "Sustaining open innovation through a "Center of Excellence"", Strategy \& Leadership, 2019.

[39] Ritchie, H. and M. Roser, "Plastic pollution", Our World in Data, 2018.

[40] Rittel, H.W.J. and M.M. Webber, "Dilemmas in a general theory of planning", Policy Sciences, 4(2), 1973, pp. 155169.

[41] Robertson, S., "Understanding inverse document frequency: on theoretical arguments for IDF", Journal of documentation, 60(5), 2004, pp. 503-520.

[42] Robinson, N.J. and C. Figgener, "Plastic straw found inside the nostril of an olive ridley sea turtle", Marine Turtle Newsletter, 147, 2015, pp. 5-6.

[43] Schryen, G., "Revisiting IS business value research: what we already know, what we still need to know, and how we can get there", European Journal of Information Systems, 22(2), 2013, pp. 139-169.

[44] Silge, J. and D. Robinson, "tidytext: Text mining and analysis using tidy data principles in $r$ ", The Journal of Open Source Software, 1(3), 2016, p. 37.

[45] Sun, J., J. Shen, S. Chen, M. Cooper, H. Fu, D. Wu, and Z. Yang, "Nanofiller reinforced biodegradable PLA/PHA composites: Current status and future trends", Polymers, 10(5), 2018, p. 505.

[46] Sun, J. and K. Yang, "The Wicked Problem of Climate Change: A New Approach Based on Social Mess and Fragmentation", Sustainability, 8(12), 2016, p. 1312.

[47] Tong, X. and D. Tao, "The rise and fall of a "waste city" in the construction of an "urban circular economic system": The changing landscape of waste in Beijing", Resources, Conservation and Recycling, 107, 2016, pp. 10-17.

[48] Wagner, M., S. Lambert, and M.W. Lambert, Freshwater microplastics, Springer International Publishing Cham, Switzerland, 2018.

[49] World Economic Forum, Ellen MacArthur Foundation, and McKinsey \& Company, The New Plastic Economy: Rethinking the future of plastics, January 2016.

[50] World Economic Forum, Ellen MacArthur Foundation, and Systemiq, The New Plastic Economy: Catalysing action, January 2017. 